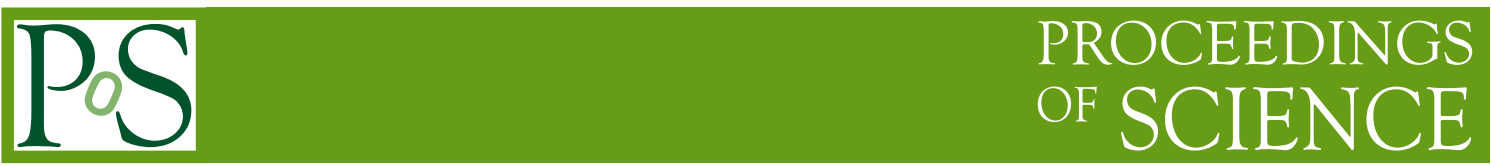

\title{
Measurements with a Si-strip telescope
}

\author{
Simone Bianco*; Max Becker, Kai-Thomas Brinkmann, Ralf Kliemt, Karsten Koop, \\ Robert Schnell, Thomas Würschig, Hans-Georg Zaunick \\ Helmholtz-Institut für Strahlen- und Kernphysik \\ E-mail: bianco@hiskp.uni-bonn. de
}

\begin{abstract}
A tracking station composed of silicon strip sensors was designed, built and tested. It is a beam telescope equipped with four boxes containing single-sided and double-sided silicon strip sensors. The boxes can be moved along the longitudinal direction and one of them can be rotated to vary the angle on incidence of the beam relative to the face of the sensor. These features allow to study in detail the effects of changes in the setup on energy loss, cluster size and resolution. The tracking station was used with particle beams at different facilities (COSY, DESY, ELSA). A selection of scatterers has been placed in the center of the station and the consequent deflection angle distributions were measured. This system allowed to tune the simulation of low-energy hadron interactions with the material of a silicon tracking detector. Furthermore, light materials such as carbon foams with different densities and compositions were characterized during these tests. Analyses of measured data were compared with Monte Carlo simulations of the experimental setup.
\end{abstract}

10th International Conference on Large Scale Applications and Radiation Hardness of Semiconductor Detectors,

July 6-8, 2011

Firenze Italy

\footnotetext{
* Speaker.

${ }^{\dagger}$ The author wants to thanks BCGS and BMBF for supporting this research work.
} 


\section{Analysis of the Bonn tracking station data}

The Bonn MVD group designed, built and tested a tracking station equipped with silicon strip sensors. It is composed of four boxes which can be positioned along a longitudinal axis spanning a range of nearly $200 \mathrm{~cm}$. Each box contains $2 \mathrm{~cm} \times 2 \mathrm{~cm}$ sensors $(1.92 \mathrm{~cm} \times 1.92 \mathrm{~cm}$ active area) with a thickness of $300 \mu \mathrm{m}$, pitch of $50 \mu \mathrm{m}$ and stereo angle of $90^{\circ}$. The sensors are designed with $\mathrm{p}$ strips in a n-bulk and they are depleted with a punch through bias at a voltage of approximately $60 \mathrm{~V}$. Two boxes are equipped with a double sided sensor, while the others include two single sided sensors each, disposed with a $90^{\circ}$ stereo angle. The boxes feature a cut out in the areas in front and behind the sensors with respect to the beam direction. These windows are covered with a 20 $\mu \mathrm{m}$ thick mylar layer, which can be neglected in the following material budget considerations. The passive elements such as front-end chips and supply boards are positioned within the boxes out of the beam occupancy. Four scintillating fingers are used to trigger the data acquisition. Each box can be positioned independently from the others and one of them can be rotated with respect to the beam direction. The tracking station is shown in Fig.1 during a beam time at the COSY [1] proton synchrotron. The data acquisition (DAQ) system used during the tests is summarized in Fig.2 (see [2]). The analog signals are readout from each strip of the sensors with APV25 front-end ASICs. The analog output is sampled with an ADC module, connected to the frontend chip over a twisted-pair cable, which is plugged into FPGA modules where online baseline compensation, pedestal tracking and cluster finding are carried out. The acquired data is finally stored into ROOT[3] files. The system was tested to be fully functional with rates up to of some thousands of events per second.

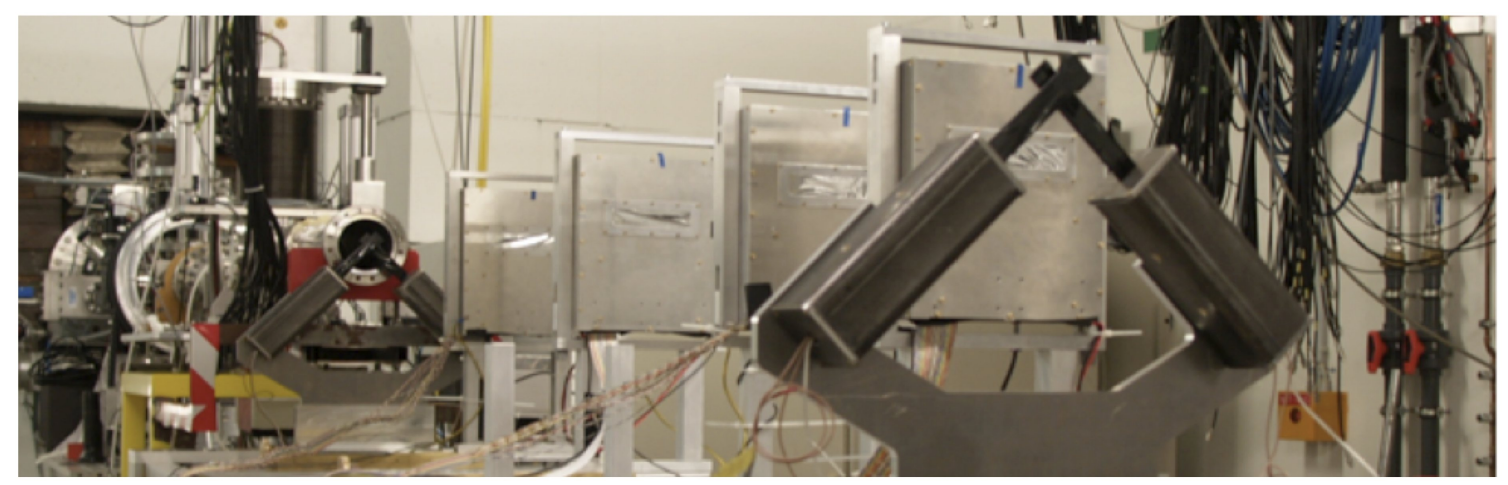

Figure 1: The Bonn tracking station set up at the COSY accelerator (Jülich).

The tracking station has been tested in different beam conditions:

- $2.95 \mathrm{GeV} / \mathrm{c}$ and $0.89 \mathrm{GeV} / \mathrm{c}$ protons at $\mathrm{COSY}$;

- 1 to $5 \mathrm{GeV}$ electrons at DESY [4];

- Bremsstrahlung photons from an electron beam of $2 \mathrm{GeV}$ at ELSA [5];

- $e^{+} e^{-}$pair production from Bremsstrahlung photons at ELSA. 


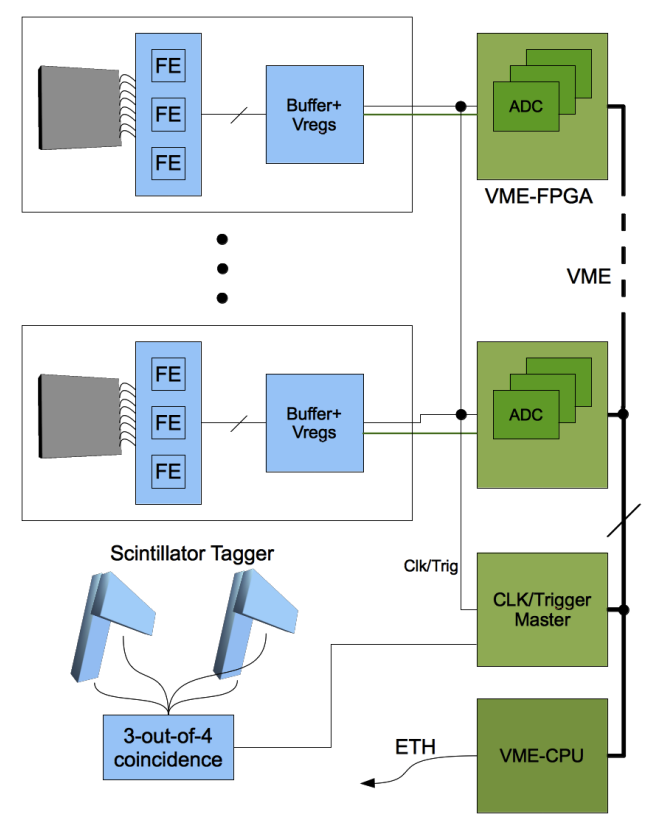

Figure 2: Structure of the DAQ system used during the beam tests.

\section{Analysis tools}

The analysis of the data measured with the tracking station was performed within the PANDA [6] simulation and reconstruction software framework [7]. A custom software infrastructure (see Fig. 3(a)) is used to import the raw data, to perform clustering and hit reconstruction, to realize an offline alignment of the sensors together with a channel by channel energy loss calibration (see also [8]). The final analysis of the results is then performed within this framework. This set of software tools consists of C++ ROOT-based classes. The offline alignment is realized with an iterative procedure based on the exclusive residual distributions of each sensor. The coordinates

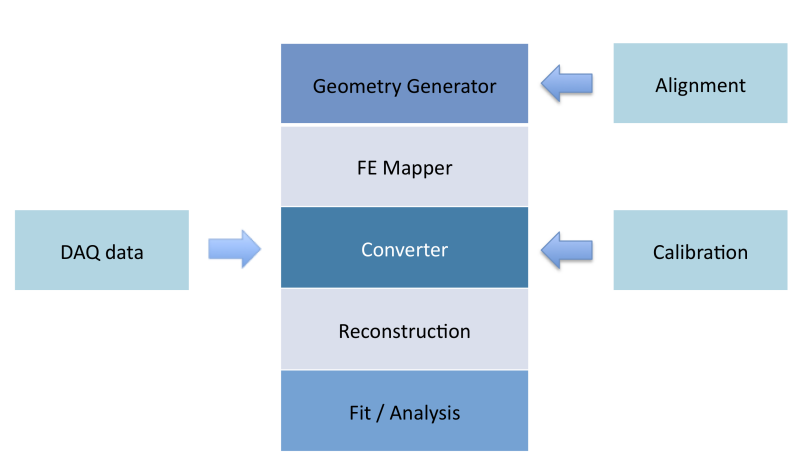

(a) Structure of the analysis tools

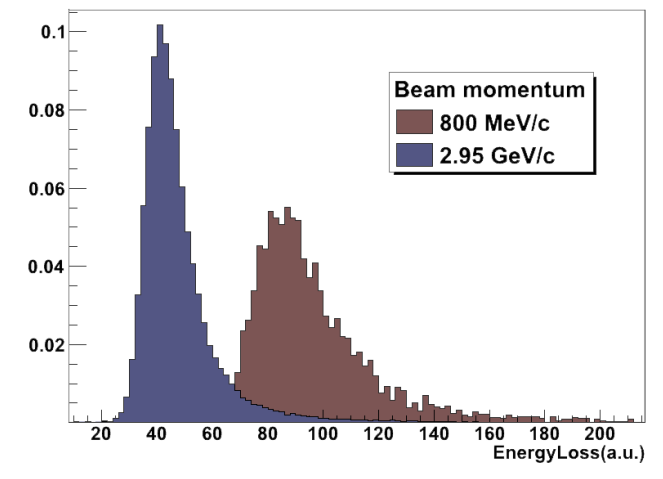

(b) Energy loss measured in one sensor with beams of protons of two different momenta

Figure 3: Analysis infrastructure and results from the measurements. 
of the position of each sensor are adjusted iteratively in order to obtain a residual distribution with the mean as close as possible to zero. The whole procedure is repeated until the target precision is reached. The final positions of the sensors are defined in a corrected geometry, which is then used for the reconstruction and the analysis. The ratio between ADC counts and the expected energy loss (given the beam energy and the geometry) is used to set the energy calibration for each channel of the sensors. This operation can be performed in the software supplying the raw data converter with a calibration map consisting of a list of the ratios to be used to obtain a correct energy calibration. Figure 3(b) shows a comparison of the energy loss distributions measured in one sensor with proton beams of different momenta.

\section{Rotation and shift of one sensor}

One of the boxes can be rotated with respect to the beam direction. This allows changing the incident angle of the beam, modifying the average cluster size and the energy lost inside the detector. Systematic measurements were performed with the setup shown in Fig.4 and compared to simulations realized within the PANDA framework, utilising the same clustering algorithm and analysis tools used to treat measured data. This allows a direct comparison with measured data. Fig.5 shows

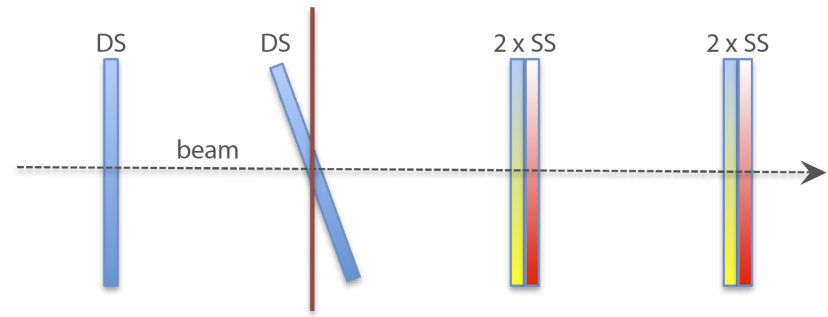

Figure 4: Top view of the setup used for the measurements performed rotating one double sided sensor. Double sided sensors are labeled with DS, while single sided ones with SS.
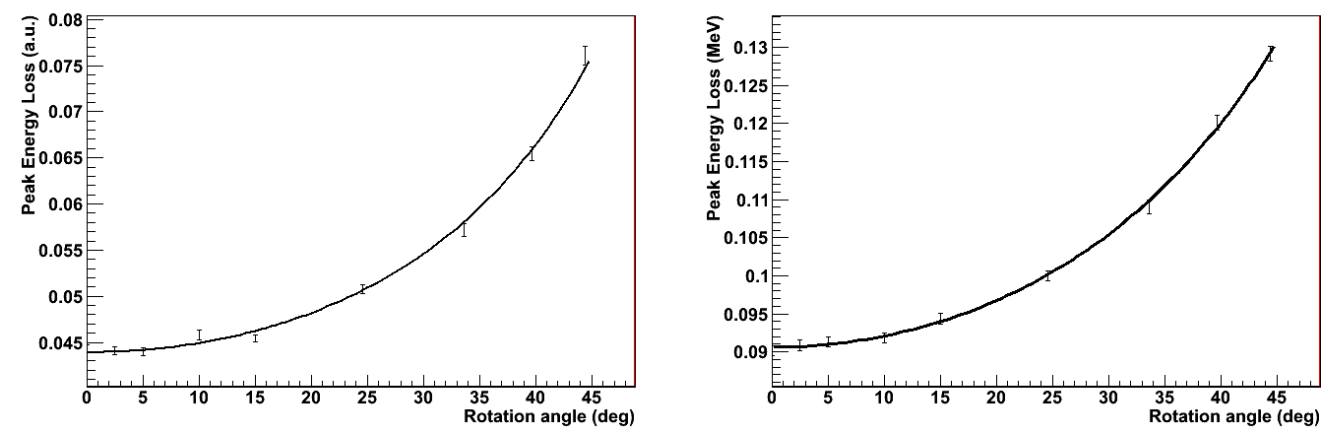

Figure 5: Peak energy loss of $4 \mathrm{GeV}$ electrons measured in one sensor as a function of the rotation angle of the module with respect to the beam direction (left) and simulation of the same setups (right).

the results obtained with $4 \mathrm{GeV}$ electrons. The energy loss inside one sensor increases with bigger incident angles and the measured trend is compatible with the one predicted by simulations. The 
cluster size, defined by the number of strips included in the cluster, was also studied. A comparison with simulation is shown in Fig.6(a) and Fig.6(b). Both data and simulations show a bump of the cluster size for a rotation of about $9^{\circ}$. Such angle corresponds to crossing the full pitch of a strip, while crossing the full thickness of the sensor: $\alpha=\arctan \left(\frac{\text { pitch }}{\text { thickness }}\right)=\arctan \left(\frac{50 \mu \mathrm{m}}{300 \mu \mathrm{m}}\right)=9.46^{\circ}$. At larger angles, the deposited energy is shared among strips, at smaller angles the sensor appears thinner. Though some quantitative differences are present, the trends of measured data and simulation are in agreement. The discrepancies are due to threshold settings in the simulations, which cannot be tuned exactly on the hardware ones since the absolute ADC energy loss calibration is not taken into account at the moment. In particular the cut on the minimum charge which distinguishes small signals from noise has a significant impact on cluster sizes, since this directly reflects in the minimum charge which can be collected by a single strip when it belongs to a reconstructed cluster. A higher threshold reduces the cluster size, not taking into account strips with a small collected charge (see Fig. 6(c)).

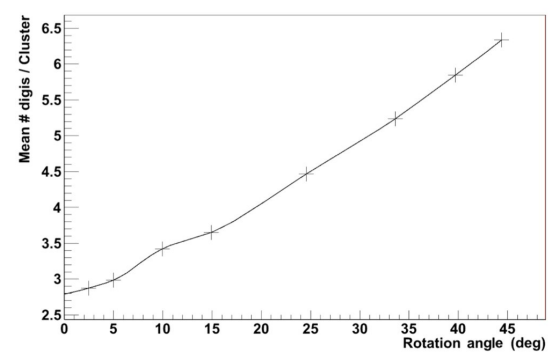

(a) Measurements of the cluster size.

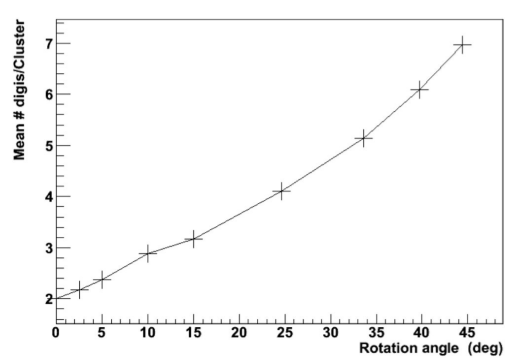

(b) Simulation of the cluster size.

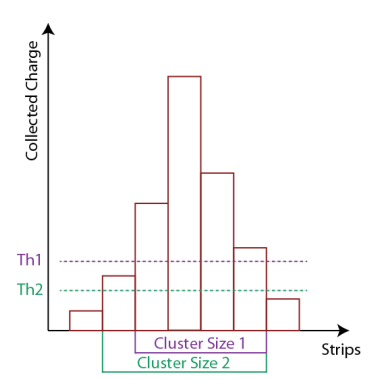

(c) Effect of thresholds on the cluster size.

Figure 6: Cluster size in terms of strips: measurements, simulation and threshold effects.

The four boxes can be moved along the beam direction. A scan was performed with a beam of $3 \mathrm{GeV}$ electrons. Changing the position of the second box, the effect on the resolution of the tracking station was studied. The unbiased resolution ${ }^{1}$ was chosen as a first estimator for the track resolution. It represents the geometrical mean of the exclusive residuals calculated on each sensor. Measurements summarized in Fig.7 show that the best unbiased resolution is obtained when the second box is positioned as close as possible to the first one. At this beam energy both sensors and the air between the boxes are not negligible in terms of material budget, therefore they influence the resolutions obtained with different configurations of the tracking station.

\section{Resolution estimations}

Simulations were performed to estimate the resolution reachable with different configurations of the tracking station. Here the aim was to study the performance of the telescope used to characterize other detectors. Therefore an ideal detector (with perfect resolution, $\sigma=0$ and realized with a $300 \mu \mathrm{m}$ thick silicon square) was placed at the center of the station. The width of the residual

\footnotetext{
${ }^{1} R E S_{x}=\sqrt[4]{\sigma_{1}^{x} * \sigma_{2}^{x} * \sigma_{3}^{x} * \sigma_{4}^{x}}$, where $\sigma_{i}^{x}$ is the standard deviation of the distribution of exclusive x-residuals for the $i^{\text {th }}$ sensor.
} 

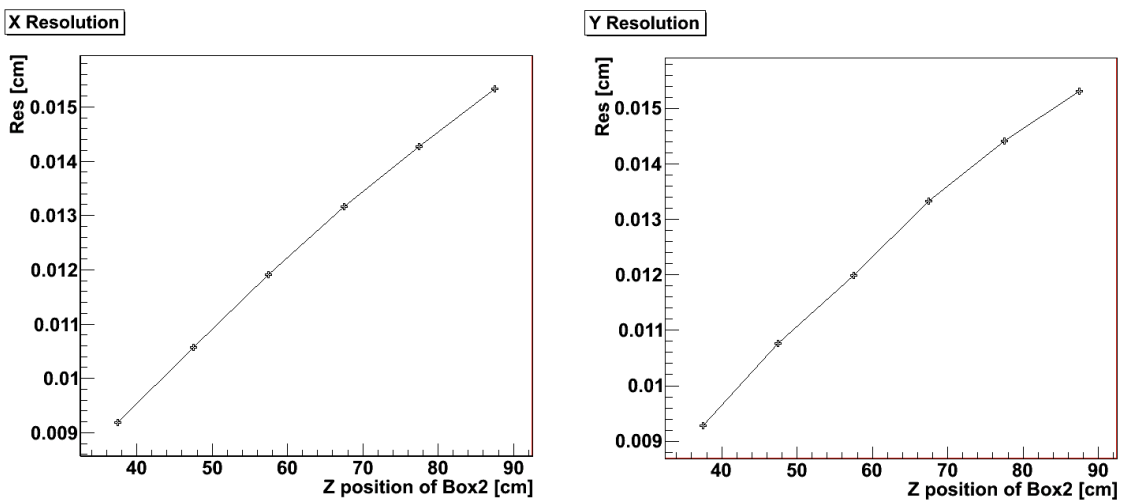

Figure 7: Results of the longitudinal scan performed at DESY with $3 \mathrm{GeV}$ electrons. The second box was moved along the tracking station in a $50 \mathrm{~cm}$ wide range. The figures show the estimator previously defined as a function of the position of the second box.

distributions calculated between the hit measured by the ideal detector and the extrapolation obtained with the tracking station points was used as estimator of the resolution for these studies. A beam of $5 \mathrm{GeV}$ electrons was propagated with different geometries of the telescope, changing the longitudinal positions of the four boxes. Table 1 summarizes the configurations used for the sim-

\begin{tabular}{|c|c|c|c|c|c||c|c|}
\hline & $\mathrm{B} 1$ & $\mathrm{~B} 2$ & Device & $\mathrm{B} 3$ & $\mathrm{~B} 4$ & $\Delta_{x}$ & $\Delta_{y}$ \\
\hline & $\mathrm{z}(\mathrm{cm})$ & $\mathrm{z}(\mathrm{cm})$ & $\mathrm{z}(\mathrm{cm})$ & $\mathrm{z}(\mathrm{cm})$ & $\mathrm{z}(\mathrm{cm})$ & $\mu \mathrm{m}$ & $\mu \mathrm{m}$ \\
\hline \hline $\mathrm{A}$ & 16. & 86. & 110. & 145. & 185.5 & 56 & 53 \\
\hline $\mathrm{B}$ & 90. & 100. & 110. & 120. & 130. & 16 & 16 \\
\hline $\mathrm{C}$ & 65. & 85. & 110. & 139. & 159. & 34 & 34 \\
\hline
\end{tabular}

Table 1: The position of the fours boxes and of the ideal sensor are shown together with the results of the simulations performed. $\Delta_{\mathrm{x}}$ and $\Delta_{\mathrm{y}}$ are the widths of the exclusive $\mathrm{x}$ and $\mathrm{y}$ residual distributions obtained with the ideal detector. The fits were based on the four hits measured with the tracking station sensors.

ulations: in case A the boxes were distributed equidistantly along the whole length of the tracking station, in case B the sensors were positioned as close as possible to the ideal detector and case $\mathrm{C}$ is the most compact setup achievable with the actual hardware restrictions. Table 1 shows the width of the residual distributions obtained with the different setups. These results confirm what was shown in the previous section: in this energy range the best resolutions are obtained choosing the setup least affected by multiple scattering, so with small distances between the sensors and the plane where the residuals are calculated.

\section{Scattering measurements}

The tracking station was used to measure multiple scattering inside volumes of carbon-based materials. The aims of these measurements were the characterization of some materials foreseen for the support structures of the PANDA MVD and the validation of the simulation framework as a valid tool for the evaluation of the effect of passive materials inside the MVD. Beams of protons 
(at COSY) and electrons (at DESY) were casted to the scatterers, measuring the deflection induced by the multiple scatterings. The setup (shown in Fig.8) allowed to measure two hits before and two

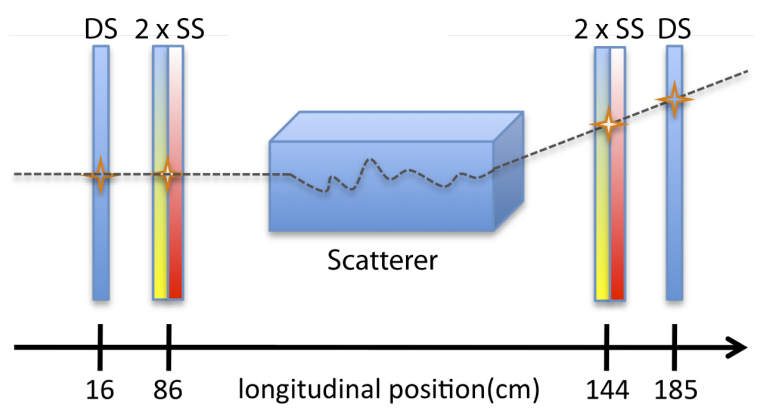

Figure 8: Setup used to measure the multiple in different carbon volumes. One double-sided strip sensor and two single-sided ones were used both before and behind the volumes.

after the scatterer. Different samples of materials were used: full carbon slabs, volumes of carbon foam and a carbon-foam based prototype of support structure for the MVD pixel disks. The last one consist of a $4 \mathrm{~mm}$ thick carbon foam half-disk, in which three steel cooling pipes with a $2 \mathrm{~mm}$ outer diameter were embedded. For each setup the projected scattering angle distributions have

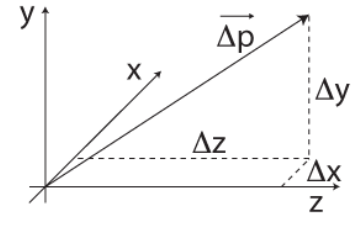

$$
\left|\overrightarrow{p_{i}}\right|=|\vec{p} f|=1
$$

$$
\theta \mathrm{x}=\arctan (\Delta \mathrm{x} / \Delta \mathrm{z})
$$

$\theta \mathrm{y}=\arctan (\Delta \mathrm{y} / \Delta \mathrm{z})$

$$
\overrightarrow{\Delta p}=\frac{\overrightarrow{p_{f}}-\overrightarrow{p_{i}}}{\left|\overrightarrow{p_{f}}-\overrightarrow{p_{i}}\right|}
$$

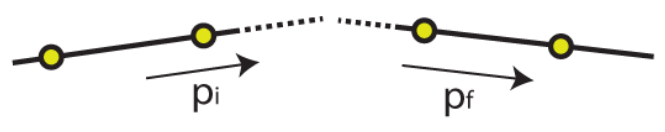

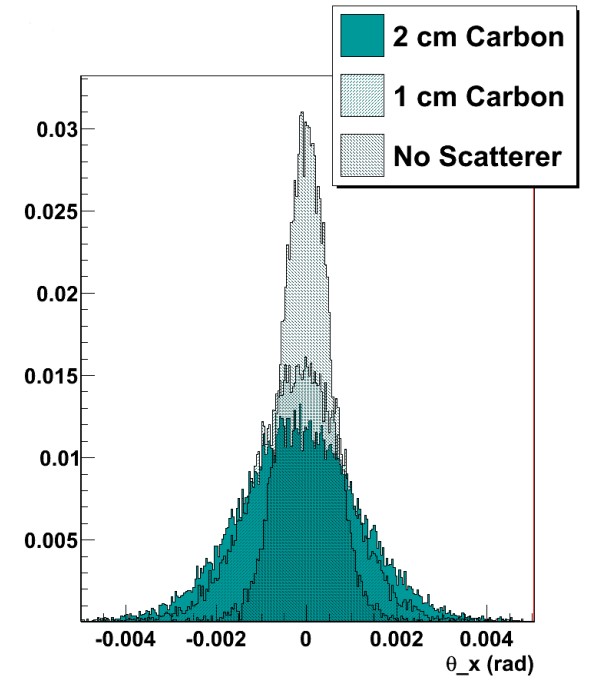

Figure 9: Distributions of the projected scattering angles (defined as described in the left part of the figure) obtained with $2.95 \mathrm{GeV} / \mathrm{c}$ protons crossing the previously described scattering volumes.

been measured (some examples are shown in Fig.9) and simulated using Geant3 (see [9]) within the PANDA framework. The obtained distributions are well described by Gaussians in their central part, so the standard deviation of their Gaussian fit was chosen as observable of interest. The results of Table 3 show that it was possible to notice the presence of light scatterers (such as the carbonfoam based support prototype) and also to verify the effect of small changes in the beam momenta. The results of the measurements performed with protons are nicely compatible with the predictions 
of simulations. Using electron beams the simulations seem to slightly overestimate the effect of the

\begin{tabular}{|c|c|c|c|c||c|c|c|c|c|}
\hline Vol. & Ptc & Mom. & Meas. $\sigma$ & Sim. $\sigma$ & Vol. & Ptc & Mom. & Meas. $\sigma$ & Sim. $\sigma$ \\
\hline & & GeV/c & $\mathrm{mrad}$ & $\mathrm{mrad}$ & & & $\mathrm{GeV} / \mathrm{c}$ & $\mathrm{mrad}$ & $\mathrm{mrad}$ \\
\hline \hline Air & $p^{+}$ & 2.95 & 0.532 & 0.536 & Air & $e^{-}$ & 5.4 & 0.243 & 0.284 \\
\hline $1 \mathrm{~cm} \mathrm{C}$ & $p^{+}$ & 2.95 & 1.02 & 1.01 & Foam & $e^{-}$ & 1.0 & 2.18 & 2.54 \\
\hline $2 \mathrm{~cm} \mathrm{C}$ & $p^{+}$ & 2.95 & 1.34 & 1.33 & Supp. Prot. & $e^{-}$ & 1.0 & 1.76 & 1.87 \\
\hline Air & $e^{-}$ & 1.0 & 1.24 & 1.40 & Supp. Prot. & $e^{-}$ & 3.0 & 0.601 & 0.611 \\
\hline Air & $e^{-}$ & 3.0 & 0.423 & 0.476 & Supp. Prot. & $e^{-}$ & 4.0 & 0.471 & 0.483 \\
\hline
\end{tabular}

Table 2: Comparison between the widths of Gaussian fits on the distributions of the projected scattering angles obtained with real measurements and Geant 3 simulations using different scatterers.

scatterers with a disagreement in the order of some $10 \%$. The scaling with beam momenta changes and radiation length of the scattering volumes is nevertheless well reproduced by the simulations.

\begin{tabular}{|c|c|c|}
\hline Vol. & Thickness & $X / X_{0}$ \\
\hline Air & $\sim 60 \mathrm{~cm}$ & 0.002 \\
\hline $1 \mathrm{~cm} \mathrm{C}$ & $1 \mathrm{~cm}$ & 0.042 \\
\hline $2 \mathrm{~cm} \mathrm{C}$ & $2 \mathrm{~cm}$ & 0.080 \\
\hline Carbon Foam & $2.5 \mathrm{~cm}$ & 0.030 \\
\hline Supp. Prot. & $4 \mathrm{~mm}$ & 0.010 \\
\hline
\end{tabular}

Table 3: Thickness in term of radiation lengths of the different scatterers. Since the volumes had slightly different porosity, the density used to calculate these values was measures for each sample.

\section{References}

[1] COSY, Jülich, Germany, http://www2.fz-juelich.de/ikp/cosy/en/.

[2] R. Schnell et al. FPGA-based readout for double-sided silicon strip detectors. JINST 6 (2011)C01008.

[3] I. Antcheva et al., ROOT - A C++ framework for petabyte data storage, statistical analysis and visualization, Computer Physics Communications 180 (2009) 2499-2512

[4] DESY, Hamburg, Germany, http://adweb.desy.de/ testbeam/.

[5] ELSA, Bonn, Germany, http://www-elsa.physik.uni-bonn.de/.

[6] PANDA Collaboration, Physics Performance Report for: PANDA - Strong Interaction Studies with Antiprotons, arXiv:0903.3905v1 [hep-ex], 2009.

[7] S. Spataro. Simulation and event reconstruction inside the PandaRoot framework. J. Phys. Conf. Ser., 119, 2008.

[8] S. Bianco. Characterization of the PANDA Micro-Vertex-Detector and Analysis of the First Data Measured with a Tracking Station. IEEE Nuclear Science Symposium Conference Record, N42(278), 2010.

[9] Geant3 manual. CERN program library W5013, 1993. 